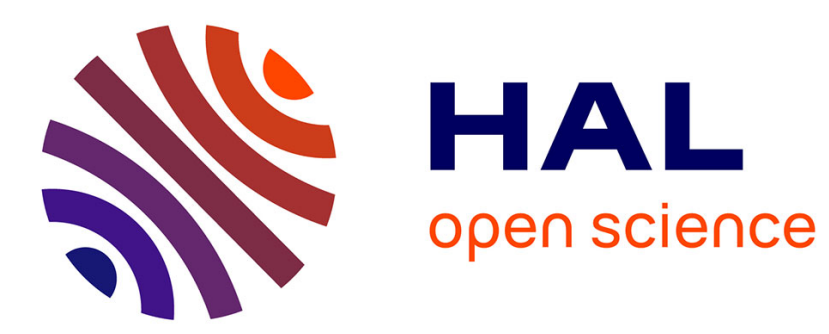

\title{
Distance Estimation in Large Immersive Projection Systems, Revisited
}

Gerd Bruder, Ferran Argelaguet Sanz, Anne-Hélène Olivier, Anatole Lécuyer

\section{To cite this version:}

Gerd Bruder, Ferran Argelaguet Sanz, Anne-Hélène Olivier, Anatole Lécuyer. Distance Estimation in Large Immersive Projection Systems, Revisited. Proceedings of IEEE Virtual Reality Conference, Mar 2015, Arles, France. hal-01149964

\section{HAL Id: hal-01149964 \\ https://hal.inria.fr/hal-01149964}

Submitted on 7 May 2015

HAL is a multi-disciplinary open access archive for the deposit and dissemination of scientific research documents, whether they are published or not. The documents may come from teaching and research institutions in France or abroad, or from public or private research centers.
L'archive ouverte pluridisciplinaire HAL, est destinée au dépôt et à la diffusion de documents scientifiques de niveau recherche, publiés ou non, émanant des établissements d'enseignement et de recherche français ou étrangers, des laboratoires publics ou privés. 


\section{Distance Estimation in Large Immersive Projection Systems, Revisited}

\author{
Gerd Bruder* \\ Department of Computer Science \\ University of Hamburg
}

\author{
Fernando Argelaguet Sanz \\ Inria Rennes
}

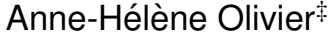 \\ Inria Rennes
}

\author{
Anatole Lécuyer ${ }^{\S}$ \\ Inria Rennes
}

\begin{abstract}
When walking within an immersive projection environment, accommodation distance, parallax and angular resolution vary according to the distance between the user and the projection walls which can influence spatial perception. As CAVE-like virtual environments get bigger, accurate spatial perception within the projection setup becomes increasingly important for application domains that require the user to be able to naturally explore a virtual environment by moving through the physical interaction space. In this paper we describe an experiment which analyzes how distance estimation is biased when the distance to the screen and parallax vary. The experiment was conducted in a large immersive projection setup with up to ten meter interaction space. The results showed that both the screen distance and parallax have a strong asymmetric effect on distance judgments. We found an increased distance underestimation for positive parallax conditions. In contrast, we found less distance overestimation for negative and zero parallax conditions. We conclude the paper discussing the results with view on future large immersive projection environments.
\end{abstract}

Index Terms: H.5.1 [Information Interfaces and Presentation]: Multimedia Information Systems-Artificial, augmented, and virtual realities; I.3.7 [Computer Graphics]: Three-Dimensional Graphics and Realism-Virtual reality

\section{INTRODUCTION}

Immersive virtual reality (VR) systems can provide users with a sense of feeling present in the displayed virtual environment (VE) similar to perceiving an environment in the real world [25]. Recent advances in hardware technologies make it possible to build immersive projection environments (IPE), such as a CAVE [7], with a large room-sized interactive workspace. Such IPEs support natural forms of interaction with the displayed virtual world in the egocentric frame of reference of the observer, including real walking. These affordances facilitate spatial impressions of the VE that are important for exploration and review in a wide range of application domains such as architecture and engineering in which users benefit from experiencing the VE at real scale.

For such applications it is essential to facilitate a veridical impression of the spatial layout, e.g., sizes, distances, and interrelations, within the perceived virtual world. Modern real-time rendering systems can create compelling immersive experiences offering most of the spatial visual cues we can find in the real world, including perspective, interposition, lighting, and shadows [27]. However, distance and size perception are often biased in such environments, causing users to over- or underestimate spatial relations [14, 18, 28]. The particular factors influencing a user's distance estimates in IPEs

\footnotetext{
*e-mail: gerd.bruder@uni-hamburg.de

$\dagger$ e-mail: fernando.argelaguet_sanz@inria.fr

†e-mail: anne-helene.olivier@inria.fr

$\S$ e-mail: anatole.lecuyer@inria.fr
}

are not yet clearly identified, and large portions of the observed misperception effects still cannot be explained [18, 24].

Although IPEs differ from the real world in many respects, issues with the visual rendering and display technologies have naturally been suggested as a potential cause of nonveridical spatial perception. One of the potential suspects for such misperception is the accommodation-convergence conflict [13]. In the real world, accommodation and convergence are coupled together and provide distance cues up to a distance of about 6 meters $[6,8,30]$. In stereoscopic display systems, the observer accommodates to the distance of the display surface to perceive objects without blur, whereas the convergence angle depends on parallax. Three parallax conditions are considered: negative parallax (object in front of the display), zero parallax (object on the display) and positive parallax (object behind the display) [2]. With negative or positive parallax the user's visual system is confronted with conflicting depth information and might be misguided by the accommodative information [9].

In this paper we describe an experiment in which we assess ego-centric distance perception in an IPE with an interaction space up to $10 \mathrm{~m}$. In such large IPEs, the accommodation distance, the accommodation-convergence mismatch and the angular resolution vary largely depending on where the user is standing and where virtual objects are displayed. In comparison to previous distance perception research in IPEs, which were restricted to small negative parallaxes due to limited interaction workspaces, to our knowledge, we detail the first analysis of distance perception in an IPE that supports to display virtual objects with such a large negative parallax.

Our main contributions are:

- We analyze the role of stereoscopic parallax for screen distances ranging from 1 to 9 meters.

- Our results reveal a strong asymmetric effect of screen distance and stereoscopic parallax.

The remainder of this paper is structured as follows. Section 2 presents background information on distance perception. Section 3 describes the experiment in which we assessed distance perception. Implications and guidelines are discussed in Section 4. Section 5 concludes the paper.

\section{BACKGROUND}

In this section, we summarize information about distance cues in the scope of the conducted experiment.

\subsection{Stereopsis}

The binocular configuration of human eyes provides the brain with two views of a scene from laterally separated positions at a fixed interpupillary distance (IPD). Solving the disparity correspondence problem, the brain may relate retinal image contents from the two eyes to one another, computing the distance to seen objects via triangulation. Further, when focusing on an object, the eyes need to rotate toward that object to bring it to the fovea of each retina. The convergence state of the eyes, changed by extrinsic muscle exertion, provides an absolute cue about the distance to an object. In 
a simplified setting, the distance $D_{0} \in \mathbb{R}^{+}$of an object can be computed from the user's IPD and the convergence angle $\alpha \in \mathbb{R}^{+}$[3] (see Figure 1):

$$
D_{0}=\frac{\text { IPD }}{2 \cdot \tan \left(\frac{\alpha}{2}\right)} .
$$

Stereoacuity is naturally limited with a conservative threshold of about 10 seconds of arc [20]. Considering this threshold and referring to Equation 1, the maximum distance at which stereopsis may produce usable data would be ca. $1.24 \mathrm{~km}$. However, in IPEs the angular resolution of pixels on a screen acts as an artificial cut-off to the capabilities of human vision.

\subsection{Accommodation}

The human eye can alter its optical power to hold objects at different distances into sharp focus on the retina. When an object is fixated by the eye, the ciliary muscles are adjusted such that a sharp image is perceived on the retina. The state of the ciliary muscles provides an absolute cue about the distance to the focused object. However, full accommodation response depends on the accommodative stimulus, i. e., responses of ciliary muscles differ between fuzzy and high-contrast stimuli [10].

The role of accommodation in distance perception is controversial. While a body of early work found negligible effects of accommodation on human distance perception, many of these results nowadays are attributed to confounds in the experimental designs (cf. [10]). While accommodation can be leveraged as a distance cue, the influence of the accommodative information declines with age [21], distance [8] and with decreasing cue reliability [13].

\subsection{Accommodation-Convergence Conflict}

Oculomotor responses of accommodation and convergence usually co-vary to provide a sharply focused view. Accommodation is mainly driven by retinal blur (monocular cue), and convergence by retinal disparity (binocular cue). However, with stereoscopic displays, the physiologically coupled oculomotor processes of convergence and accommodation are dissociated. Observers fixate an object with the same convergence as in natural viewing, but the eyes focus on the screen and not the object, which can bias distance estimation and can cause visual discomfort [13]. Loomis and Knapp [18] and Renner et al. [24] provide thorough reviews of the literature on effects of visual conflicts on distance estimation in IPEs. Although the reported studies used different materials and methods, their results agree that users tend to underestimate ego-centric distances in vista space in IPEs $[1,12,16]$. In particular, Piryankovad et al. [22] observed distance underestimation over multiple immersive large screen displays, as well as an interaction effect with the distance to the displayed target.

We have to note that most of these studies focused on virtual objects with positive parallax. In such situations, longer distances to objects correlate with smaller convergence angles, but also with reduced angular resolution and diminished accommodation responses due to more blur in retinal images. In contrast, objects displayed with negative parallax cause the conflict to reverse its sign, and accommodation responses benefit from the reduced blur in retinal images [4]. Considering that objects displayed near zero parallax approximate viewing as in the real world, it is a challenging question whether zero parallax defines a singularity of optimal distance estimation. So far, the effects of the sign of the conflict and blur in retinal images due to low angular resolution are not yet clear.

\section{PSYChOPHYSICAL EXPERIMENT}

In this section we describe the experiment which we conducted to analyze the interrelations between the ego-centric distance to the projection screen (i. e., screen distance $D_{s} \in \mathbb{R}^{+}$) and the distance to a visual target (i.e., target distance $D_{t} \in \mathbb{R}^{+}$) in terms of distance judgments measured with a triangulated pointing method [16].

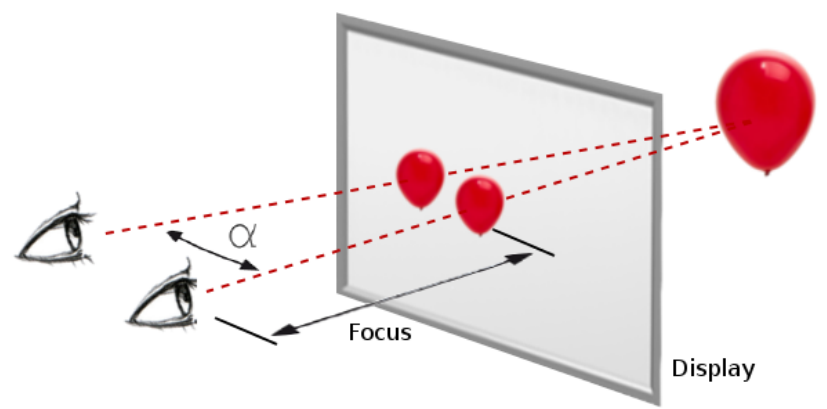

Figure 1: Illustration of object distance as a function of eye convergence angle $\alpha$ and screen distance from accommodation with stereoscopic displays.

\subsection{Materials}

The experiment was conducted in a $9.6 \mathrm{~m} \times 3 \mathrm{~m} \times 3.1 \mathrm{~m}$ (width, depth, and height) 4-sided IPE (see Figure 2) equipped with 16 Barco Galaxy projectors at 15MPixels resolution in total. The pixel size for the side walls was $1.56 \mathrm{~mm} \times 2.56 \mathrm{~mm}$ and for the front wall $1.36 \mathrm{~mm} \times 1.47 \mathrm{~mm}$. For visual display, system control and logging we used a cluster of 7 HP Z400 with $1 \times 7$ Nvidia Quadro FX 5000 and 2 HP Z420 with $1 \times 2$ Nvidia Quadro 5000 graphics cards. The VE was rendered using the Unity 3D Pro game engine with the MiddleVR plugin for multi-surface rendering. Participants wore shutter glasses (Volfoni ActivEyes Pro Radiofrequency) for stereoscopic visual stimulus presentation. The shutter glasses were tracked with 6 degrees of freedom passive markers using an ART optical tracking system with 16 cameras at an update rate of $60 \mathrm{~Hz}$. An ART Flystick 2 was used for the pointing task.

\subsection{Protoco}

Participants had to judge the distance to a seen virtual target object using the method of blind triangulated pointing, which we adapted to the configuration of our projection setup. Similar to previously introduced procedures $[11,16]$, participants held the Flystick as they observed the object. When participants were ready to judge the distance to the object, they had to close their eyes, trigger the button of the Flystick to fade to black the virtual environment, take two steps to the left or right, and point the Flystick to the object (see Figure 2). Participants were instructed to point at the target as accurately as possible while performing the side stepping at a reasonable speed to reduce effects of decreased precision caused by changes in the remembered position of the target over time (cf. [19]). Participants received no feedback about their pointing accuracy in order to minimize the effects of perception-action motor recalibration in the response method while assessing distance perception.

The visual stimulus consisted of a virtual scene as shown in Figure 2 , i. e., an all-gray virtual world, and virtual balloons were chosen as targets for the distance estimation task. Traditional helium party balloons in the real world have a standardized size of $28 \mathrm{~cm}$, thus providing known retinal size cues. Helium balloons are one of the few objects in the real world that occur floating in mid-air (cf. [26]).

Instructions were provided on a computer screen prior to the experiment. In order to focus participants on the tasks no communication between experimenter and participant was performed during the experiment after the initial training phase, in which we ensured that participants correctly understood the task. The fourth wall was closed during the experiment using an opaque black lightshield to avoid distractions. After each trial a new starting position was shown on the floor of the IPE to determine the start position and orientation of the next trial. We instructed participants always to point to the center axis of the virtual balloon with an outstretched 
arm with their dominant hand. The round shape of the target balloon has the benefit that pointing towards its center is independent of the pointing angle, i. e., rotationally invariant, which would be confounded using a traditional flat target.

\subsection{Methods}

We followed a repeated measures within-subjects design. The independent variables were the screen distance $\left(D_{s}\right)$ and the distance towards the virtual object $\left(D_{t}\right)$. We instructed participants to assume different positions while standing upright $\left(D_{s}\right)$. These positions were at $1 \mathrm{~m}$ to $9 \mathrm{~m}$ distance in $2 \mathrm{~m}$ steps from a side wall of the IPE. Virtual target objects were rendered at approximate eye height at distances of $1 \mathrm{~m}$ to $9 \mathrm{~m}$ in $2 \mathrm{~m}$ steps $\left(D_{t}\right)$. To avoid bias, each target was displayed either on the left or right side wall of the projection setup; the order was counterbalanced. During each trial users stood at a fixed distance $D_{s} \in\{1,3,5,7,9\} \mathrm{m}$ to a projection wall, which defines the screen distance, while virtual target objects were placed at a fixed distance $D_{t} \in\{1,3,5,7,9\} \mathrm{m}$ from the participant. In particular, for each screen distance we tested one condition in which the virtual target object was centered around zero parallax, i. e., where $D_{t}=D_{s}$. Participants were guided to the positions in the immersive setup via virtual markers that we projected on the floor between trials.

The experiment was divided into two main blocks: In the first block, participants performed the triangulated blind pointing task (eyes-closed block), while in a second block, we measured the ability of participants to accurately and precisely point to the 3D targets (eyes-opened block). Therefore, participants had to complete the triangulated pointing trials with open eyes, i. e., they observed a distant object, performed two side-steps, and pointed at its position without closing their eyes. We measured this ground truth pointing data to analyze pointing behavior and to calibrate the results of the first part of the experiment.

The screen distances were balanced using a Latin Squared design. For each screen distance the order of the distances towards the virtual object was randomized. For each combination, there were 4 repetitions (two at each side wall). In summary, participants completed 5 (screen distances) $\times 5$ (target distances) $\times 2$ (side walls) $\times 2$ (repetitions) $\times 2$ (experiment bocks) $=200$ trials, as well as 5 training trials for each block of the experiment, which were excluded from the analysis. Participants were allowed to take a short break at any time between trials. A short break between the two blocks of the experiment was mandatory.

The dependent variable was the distance estimate. From the initial view direction to the target object, as well as the position and pointing direction after the participant performed the side-steps, we computed the judged distance to the perceived position of the virtual target $[11,16]$.

Considering the previous results in the literature $[1,12,16]$ and the distance cues described in Section 2, our hypotheses were:

H1 No underestimation nor overestimation of the distance to objects at zero parallax.

H2 Underestimation of the distance to objects exhibiting positive parallax.

H3 Overestimation of the distance to objects exhibiting negative parallax.

H4 More accurate distance estimation for longer screen distances.

Furthermore, we collected demographic information with a questionnaire before the experiment and measured the participants' sense of presence with the Slater-Usoh-Steed (SUS) questionnaire [29], as well as simulator sickness with the Kennedy-Lane

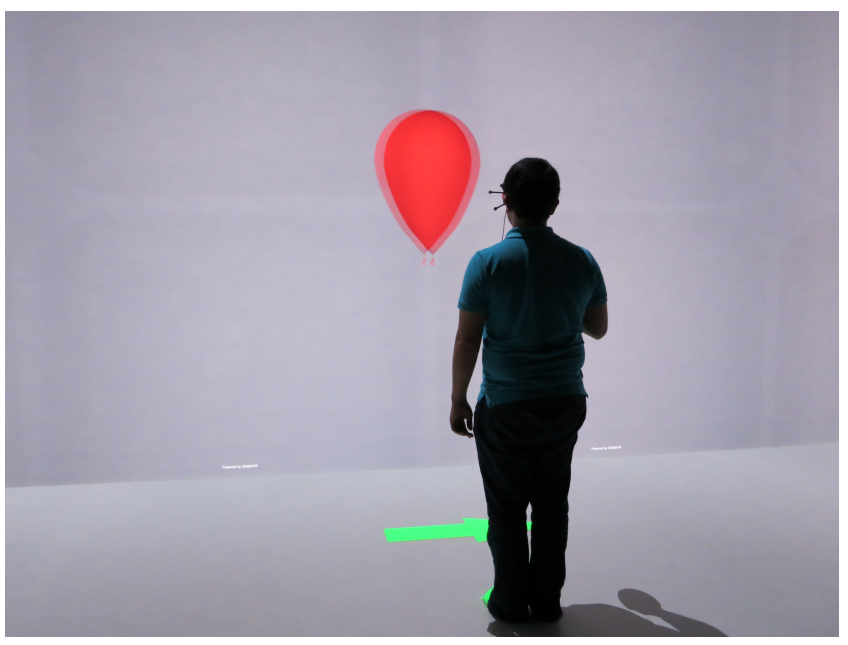

Figure 2: Photo of a participant during the experiment while performing the blind triangulated pointing task.

SSQ [15] before and after the experiment. The total time per participant including pre-questionnaires, instructions, training, experiment, breaks, and debriefing was 1 hour. Participants were immersed in the VE for about 45 minutes.

\subsection{Participants}

We recruited 15 participants for our experiment, 13 male and 2 female (aged from 23 to $38, M=28.1$ ). The participants were students or professionals in computer science or engineering. All participants reported that they were right-handed, which we confirmed with the Lateral Preference Inventory questionnaire [5]. Six participants wore glasses and three wore contact lenses during the experiment. We measured each participant's visual acuity before the experiment using a Snellen chart. 13 participants had at least $20 / 20$ visual acuity and 2 participants had 20/30. None of the participants reported known vision disorders, such as color or night blindness, dyschromatopsia, or a known displacement of balance. 13 participants reported previous experience with 3D stereoscopy (rating scale $0=$ yes, $4=$ no, $\mathrm{M}=1.67, \mathrm{SD}=1.45$ ). 10 participants had participated in a study in the immersive projection setup before. Using the technique proposed by Willemsen et al. [30] we measured the IPD of each participant before the experiment started $(\mathrm{M}=6.49 \mathrm{~cm}, \mathrm{SD}=.29 \mathrm{~cm})$. Moreover, we measured the eye height of each participant $(\mathrm{M}=1.65 \mathrm{~m}, \mathrm{SD}=.063 \mathrm{~m})$.

The data from one of the users was not considered in the analysis due to a technical problem during the experiment.

\subsection{Results}

First, we analyzed the results of the eyes-opened block. We observed angular errors of $\mathrm{M}=0.45$ degrees ( $\mathrm{SD}=1.19$ degrees) in pointing performance with eyes open. The results show that participants achieved overall high pointing accuracy and precision in the considered range of target distances with no significant bias. The results show that the system was working properly and the protocol is valid.

Regarding the eyes-closed block, Figures $3 \mathrm{a}$ and $3 \mathrm{~b}$ show the pooled results for the screen distances $D_{s} \in\{1,3,5,7,9\} \mathrm{m}$ with the standard error of the mean. We found no significant difference between the results for the left and right side wall of the immersive projection setup, so we pooled the responses. The $x$-axes show the actual target distances $D_{t} \in\{1,3,5,7,9\} \mathrm{m}$, the $y$-axes show the judged target distances. The gray lines show the distribution of judged distances $D_{j} \in \mathbb{R}_{0}^{+}$in the different conditions. We computed 


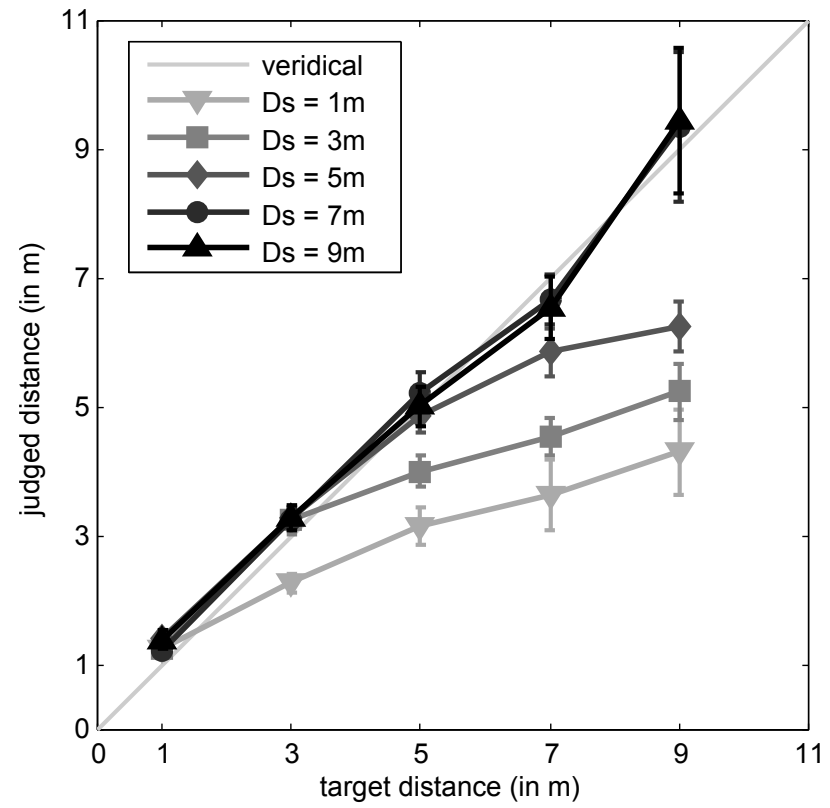

(a)

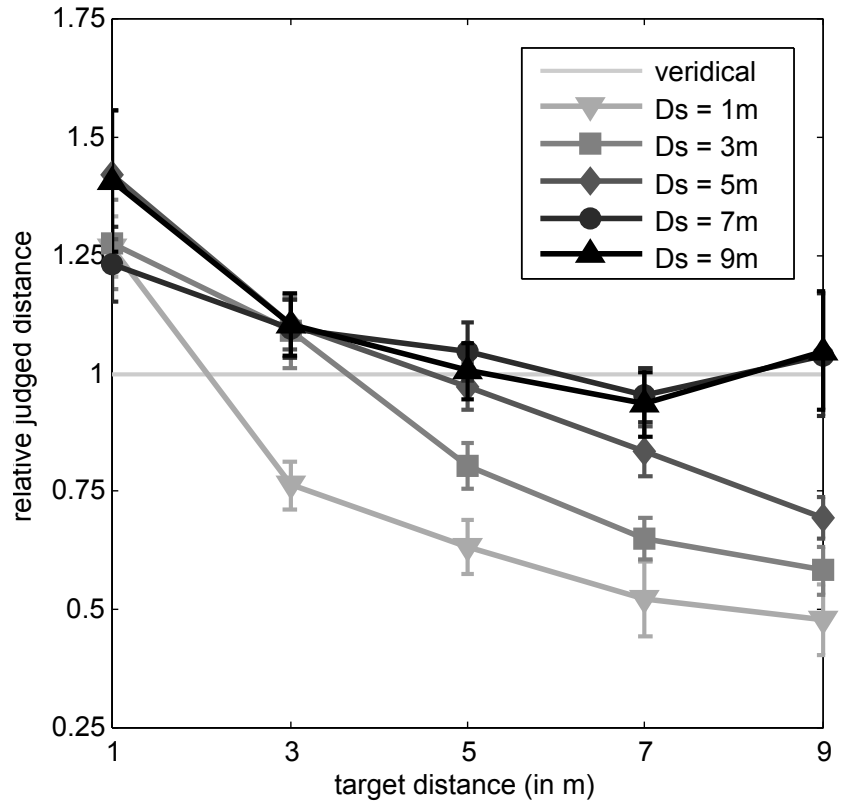

(b)

Figure 3: Pooled results of the judged distances for the different screen distances $\left(D_{s}\right)$ in the experiment. The $x$-axes show the actual distance to the target object. The $y$-axes show the (a) absolute and (b) relative judged distance. The light to dark gray lines show the results for $D_{s} \in\{1,3,5,7,9\}$ meters.

relative judged distances as $D_{j} / D_{t}$, i. e., values near 1.0 indicate ideal results, whereas values $>1$ indicate overestimation, and values $<1$ underestimation.

The results were normally distributed according to a ShapiroWilk test at the $5 \%$ level. We analyzed the results with a repeatedmeasures ANOVA and Tukey multiple comparisons at the 5\% significance level. Degrees of freedom were corrected using Greenhouse-Geisser estimates of sphericity when Mauchly's test indicated that the assumption of sphericity had been violated. In the following, we report statistics for both absolute and relative judgments to account for the two mainly used types of analyses in the distance estimation literature.

We found a significant main effect of screen distance on absolute distance judgments $\left(F(1.99,25.84)=27.367, p<.001, \eta_{p}^{2}=.678\right)$ and on relative distance judgments $(F(2.11,27.40)=22.749$, $p<.001, \eta_{p}^{2}=.636$ ). Post-hoc tests showed that the judged distances between each two screen distances were significantly different $(p<.05)$, except between $D_{s}=5 \mathrm{~m}$ and $D_{s}=7 \mathrm{~m}$, and between $D_{s}=7 \mathrm{~m}$ and $D_{s}=9 \mathrm{~m}$. In addition, we found a significant main effect of target distance on absolute distance judgments $\left(F(1.30,16.87)=67.094, p<.001, \eta_{p}^{2}=.838\right)$ and on relative distance judgments $\left(F(1.31,17.02)=21.933, p<.001, \eta_{p}^{2}=.628\right)$. Post-hoc tests showed that the judged distances between each two target distances were significantly different $(p<.05)$, except between $D_{t}=7 \mathrm{~m}$ and $D_{t}=9 \mathrm{~m}$. Moreover, we found a significant interaction effect between screen distance and target distance on absolute distance judgments $\left(F(16,208)=10.499, p<.001, \eta_{p}^{2}=.447\right)$ and on relative distance judgments $(F(16,208)=5.819, p<.001$, $\left.\eta_{p}^{2}=.309\right)$. Post-hoc tests showed, objects exhibiting zero and negative parallax exhibited similar distance estimations for each $D_{s}$. For example, at $D_{t}=1$, there are no significant differences among each $D_{s}$, this is also true for $D_{t}=3$ when $D_{s} \geq 3$, for $D_{t}=5$ when $D_{s} \geq 5$, and for $D_{t}=7$ when $D_{s} \geq 7$. The only exception is $D_{t}=9$ in which there is no significant difference among $D_{s}=7$ and $D_{s}=9$. In con- trast, for objects exhibiting positive parallax, post-hoc tests showed significant differences (all $p<.05)$ among all screen distances.

Finally, we analyzed the results considering the difference between target and screen distances $\left(D_{t}-D_{s}\right)$. Figure 4 shows the pooled data. We compared pooled distance judgments for targets at zero parallax, positive parallax, and negative parallax. We observed a main effect of parallax on relative distance judgments $\left(F(2,26)=41.106, p<.001, \eta_{p}^{2}=.760\right)$. Post-hoc tests showed that relative distance judgments were significantly closer to veridical for zero parallax than for positive parallax $(t(13)=8.849, p<.001)$. Moreover, relative distance judgments significantly differed between positive and negative parallax $(t(13)=6.833, p<.001)$. We found a trend in relative distance judgments between zero parallax and negative parallax $(t(13)=1.931, p=.076)$.

\section{Questionnaires}

We measured a mean SSQ-score of $\mathrm{M}=17.6(\mathrm{SD}=14.8)$ before the experiment, and a mean SSQ-score of $\mathrm{M}=24.3(\mathrm{SD}=20.2)$ after the experiment. This increase in simulator sickness symptoms was not significant $(t(13)=-1.26, p=.23)$. The mean SUS-score for the reported sense of feeling present in the $\mathrm{VE}$ was $\mathrm{M}=4.2(\mathrm{SD}=.78)$, which indicates a reasonably high level of presence [29].

\subsection{Discussion}

In line with our Hypothesis H1, we observed a singularity for objects displayed at zero parallax (see Figure 4), for which participants on average were significantly more accurate at distance judgments than for objects displayed with positive parallax. However, we only found a trend for a difference between negative and zero parallax. Supporting our hypotheses $\mathrm{H} 2$ and H3, we found that participants on average overestimated distances to objects with negative parallax, but showed an underestimation for longer distances. Furthermore, the magnitude of underestimation was higher than that of the observed overestimation (see Figure 3a). 


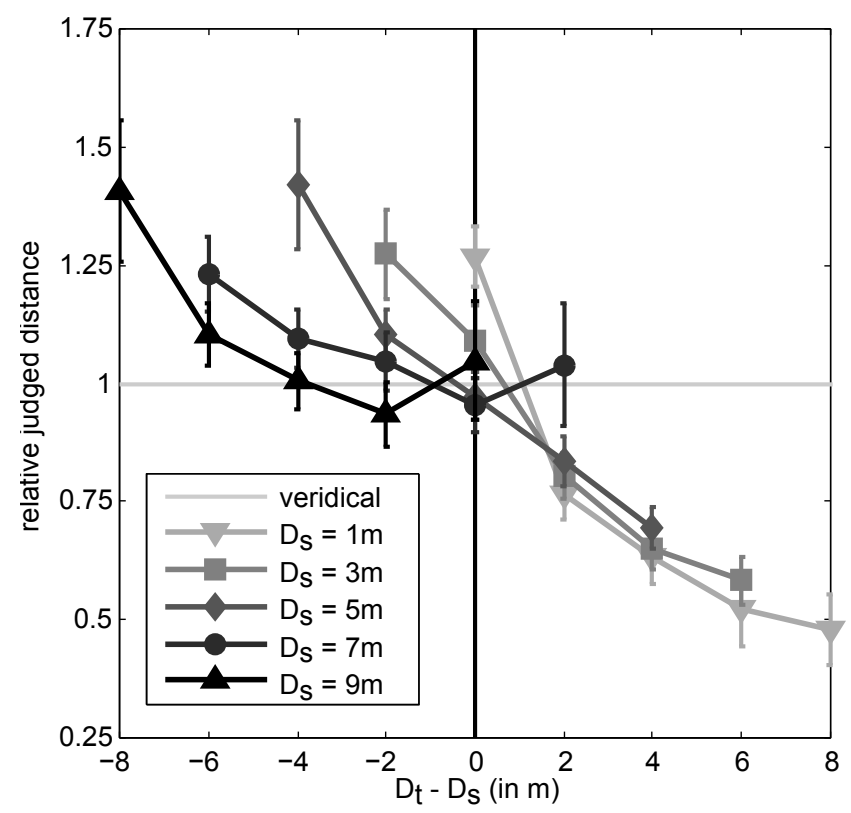

Figure 4: The $x$-axis shows the difference between target and screen distances (i.e., $D_{t}-D_{s}$ ). The $y$-axis shows the relative judged distance. The light to dark gray lines show the results for $D_{s} \in\{1,3,5,7,9\}$ meters.

The results also reveal an interaction effect between the screen distance and the distance to a virtual target object in terms of a user's distance judgments. Distance judgments are strongly affected by the position of a user in a CAVE-like immersive setup. Post-hoc tests showed that for each target distance $D_{t}$ distance judgments are similar when the virtual object exhibits zero or positive parallax $\left(D_{t} \geq D_{s}\right)$, with the only exception of $D_{t}=9$ for $D_{s}=7$ (see Figure 3a).

\section{Interpretation and Limitations}

Our results show an effect of viewing distance from the projection screen on distance judgments as well as an interaction effect with stereoscopic parallax. One possible explanation for the results is related to the accommodation-convergence mismatch. As stereoscopic parallax increases or decreases from zero parallax, the accommodation-convergence mismatch usually increases (i.e., in case of full accommodation responses, cf. Section 2), which is characterized by the convergence cue indicating the distance to the virtual object, whereas the accommodation cue indicates the distance to the physical screen. Since the difference between these indicated distances is signed, this may explain why objects with negative parallax were overestimated and objects with positive parallax underestimated. Moreover, at screen distances $D_{s} \geq 7$ depth cues from accommodation might be less taken into account for distance estimates [8], which might explain why judged distances appeared to be more accurate for longer screen distances.

However, further experimentation is needed to understand if the accommodation-convergence mismatch is the actual cause of the observed effects. There are other possible explanations for the effects: First, changes of the angular resolution may explain some of the observed underestimation. A low angular resolution may act as an artificial cut-off to binocular distance cues (see Section 2.1) and may reduce accommodation responses (see Section 2.2). The angular resolutions in our experiment ranged from $5.36 \times 8.8$ ar$\mathrm{cmin} / \mathrm{px}$ for $D_{s}=1 \mathrm{~m}$ to $0.59 \times 0.97 \mathrm{arcmin} / \mathrm{px}$ for $D_{s}=9 \mathrm{~m}$ (constant pixel size of $2.6 \mathrm{~cm} \times 1.56 \mathrm{~cm})$. The closer the user is to the screen, the lower is the angular resolution. Second, the retinal size (projection) of the virtual stimuli is proportional to the screen distance and inversely proportional to the target distance. Objects at negative parallax take up a larger screen space on the projection wall than objects at positive parallax, i. e., these objects are represented by more pixels on the wall. In the worst case scenario $D_{s}=1 \mathrm{~m}$ and $D_{t}=9 \mathrm{~m}$ the projected size is approximately $2.6 \mathrm{~cm} \times 1.5 \mathrm{~cm}$. Furthermore, the sparse depth cues in the visual stimuli in our controlled experiment might have reduced overall precision in distance judgments, and peripheral vision of the bezels of the projection setup might have had an additional effect on the results.

\section{Implications AND GUIDELINES}

The take-home-message of our experiment is that the ego-centric distance estimation of a user standing in an IPE depends to a large degree on the distance to the projection wall on which a virtual object is displayed. It generally seems to be optimal when objects are displayed centered around zero parallax, but it also seems to be acceptable when virtual objects are displayed within the physical confines of the projection walls of a CAVE-like setup. In contrast, our results show that ego-centric distances to objects that are displayed far behind a projection wall will likely be misperceived up to magnitudes of $50 \%$. As far as we know, this is the first time that these differences and asymmetric properties could be established for the tested ego-centric distances in vista space.

We suggest the following implications and guidelines for future CAVE-like setups:

- Space is the most important characteristic for distance perception in IPEs, optimally providing about $6-7 \mathrm{~m}$ distance around a user.

- Virtual objects with high demands on accurate spatial perception should be presented at zero or negative parallax.

\section{CONCLUSION AND FUtURE WORK}

In this paper we presented the first study of egocentric distance perception with view on screen distance and stereoscopic parallax in a large ten meter immersive projection setup. Our experiment reveals that the spatial impression of rendered virtual objects greatly depends on the relative position of the user with respect to the virtual objects and the projection screens.

We found a large impact of how far a user is standing away from the projection walls, and whether a virtual object is displayed within a CAVE-like setup with negative parallax or outside with positive parallax.

Distance estimation benefits from zero and negative parallaxes. Although our experiment revealed distance overestimation for close objects at negative parallaxes, the magnitude of this effect was limited compared to the large underestimation we observed for large positive parallaxes. We discussed implications and guidelines for the development of IPEs, showing that the space is the most important requirement for distance perception.

Future work should focus on the effects of negative parallax on visual fatigue and novel visualization displays. First, while distance perception benefits from larger IPEs, it should be considered that objects displayed with negative parallax are likely to increase visual fatigue as compared with positive parallax [23]. However, no studies have yet addressed the effects of negative parallax in large IPEs. Second, a possible explanation for the effects is the accommodation-convergence conflict, which is an inherent limitation of current-state stereoscopic displays, but first prototypes exist which have the potential to alleviate these conflicts in the future. In particular, light field displays [17] may provide a viable alternative to traditional displays once the supported depth range is improved and the computational complexity can be handled. 


\section{ACKNOWLEDGEMENTS}

This work was supported by the Vision Advanced Infrastructure for Research (VISIONAIR) project. Authors of this work are supported by the French National Research Agency ANR, project PERCOLATION number ANR-13-JS02-0008 and by the German Research Foundation. We thank our participants and Marwan Badawi for support with the implementation of the experiment.

\section{REFERENCES}

[1] I. V. Alexandrova, P. T. Teneva, S. de la Rosa, U. Kloos, H. H. Bülthoff, and B. J. Mohler. Egocentric distance judgments in a large screen display immersive virtual environment. In Proceedings of the Symposium on Applied Perception in Graphics and Visualization (APGV), pages 57-60. ACM, 2010.

[2] P. Bourke. Calculating Stereo Pairs (http: //paulbourke.net/ stereographics/stereorender/), 1999.

[3] G. Bruder, A. Pusch, and F. Steinicke. Analyzing effects of geometric rendering parameters on size and distance estimation in on-axis stereographics. In Proceedings of ACM Symposium on Applied Perception (SAP), pages 111-118, 2012.

[4] G. Bruder, F. Steinicke, and W. Stuerzlinger. Touching the Void Revisited: Analyses of Touch Behavior On and Above Tabletop Surfaces. Lecture Notes in Computer Science: Human-Computer Interaction INTERACT 2013, 8117:278-296, 2013.

[5] S. Coren. The Lateral Preference Inventory for measurement of handedness, footedness, eyedness, and earedness: Norms for young adults. Bulletin of the Psychonomic Society, 31(1):1-3, 1993.

[6] S. H. Creem-Regehr, P. Willemsen, A. A. Gooch, and W. B. Thompson. The influence of restricted viewing conditions on egocentric distance perception: implications for real and virtual environments. Perception, 34(2): 191-204, 2005.

[7] C. Cruz-Neira, D. J. Sandin, T. A. DeFanti, R. Kenyon, and J. C. Hart. The CAVE, Audio Visual Experience Automatic Virtual Environment. Communications of the ACM, pages 64-72, June 1992.

[8] J. E. Cutting and P. E. Vishton. Perceiving layout and knowing distances: The integration, relative potency, and contextual use of different information about depth. In W. Epstein and S. J. Rogers, editors, Handbook of perception and cognition, volume Perception of space and motion, pages 69-117. Academic Press, San Diego, CA, USA, 1995.

[9] D. Drascic and P. Milgram. Perceptual issues in augmented reality. In M. T. Bolas, S. S. Fisher, and J. O. Merritt, editors, Stereoscopic Displays and Virtual Reality Systems III, volume 2653, pages 123 134. SPIE, Bellingham, WA, USA, 1996.

[10] K. Fisher and K. J. Ciuffreda. Accommodation and apparent distance. Perception, 17(5):609-621, 1988.

[11] S. S. Fukusima, J. M. Loomis, and J. A. Da Silva. Visual perception of egocentric distance as assessed by triangulation. Journal of Expimental Psychology: Human Perreption and Pedormance, 23(1):86-100, 1997.

[12] T. Y. Grechkin, T. Dat Nguyen, J. M. Plumert, J. F. Cremer, and J. K. Kearney. How does presentation method and measurement protocol affect distance estimation in real and virtual environments? ACM Transactions on Applied Perception (TAP), 7:1-18, 2010.

[13] D. M. Hoffman, A. R. Girshick, K. Akeley, and M. S. Banks. Vergence-accommodation conflicts hinder visual performance and cause visual fatigue. Journal of Vision, 8(3):1-30, 2008.

[14] V. Interrante, B. Ries, J. Lindquist, and L. Anderson. Elucidating the Factors that can Facilitate Veridical Spatial Perception in Immersive
Virtual Environments. In Proceedings of Virtual Reality, pages 11-18. IEEE, 2007.

[15] R. S. Kennedy, N. E. Lane, K. S. Berbaum, and M. G. Lilienthal. Simulator sickness questionnaire: An enhanced method for quantifying simulator sickness. International Journal of Aviation Psychology, 3(3):203-220, 1993.

[16] E. Klein, J. E. Swan, G. S. Schmidt, M. A. Livingston, and O. G. Staadt. Measurement protocols for medium-field distance perception in large-screen immersive displays. In Proceedings of Virtual Reality $(V R)$, pages 107-113. IEEE, 2009.

[17] M. Levoy and P. Hanrahan. Light field rendering. In Proceedings of the ACM Annual Conference on Computer Graphics and Interactive Techniques (SIGGRAPH), pages 31-42, 1996.

[18] J. M. Loomis and J. M. Knapp. Visual perception of egocentric distance in real and virtual environments. In N. J. Mahwah, L. J. Hettinger, and M. W. Haas, editors, Virtual and adaptive environments, volume Virtual and adaptive environments, pages 21-46. Mahwah, 2003.

[19] C. C. Pagano, R. P. Grutzmacher, and J. C. Jenkins. Comparing verbal and reaching responses to visually perceived egocentric distances. Ecological Psychology, 13(3):197-226, 2001.

[20] S. Palmisano, B. Gillam, D. G. Govan, R. S. Allison, and J. M. Harris Stereoscopic perception of real depths at large distances. Journal of Vision, 10(6): 1-16, 2010.

[21] B. Pierscionek. In vitro alteration of human lens curvatures by radial stretching. Experimental Eye Research, 57:629-635, 1993.

[22] I. V. Piryankova, S. de la Rosa, U. Kloos, H. H. Bülthoff, and B. J. Mohler. Egocentric distance perception in large screen immersive displays. Elsevier Displays, 34(2):153-164, 2013.

[23] Z. Qian, X. Wang, C. Lan, and W. Li. Analysis of fatigue with 3D TV based on EEG. In Proceedings of the IEEE International Conference on Orange Technologies (ICOT), pages 306-309, 2013.

[24] R. S. Renner, B. M. Velichkovsky, and J. R. Helmert. The perception of egocentric distances in virtual environments - a review. ACM Comput. Surv., pages 1-38, 2013.

[25] M. Slater. Place illusion and plausibility can lead to realistic behaviour in immersive virtual environments. Philosophical Transactions of the Royal Society (B), 364(1535):3549-3557, 2009.

[26] W. Stuerzlinger, D. Dadgari, and J.-Y. Oh. Reality-based object movement techniques for 3D. In Proceedings of ACM CHI Workshop: What is the Next Generation of Human-Computer Interaction?, pages 1-4. ACM, 2006.

[27] W. Thompson, R. Fleming, S. Creem-Regehr, and J. K. Stefanucci. Visual Perception from a Computer Graphics Perspective. A. K. Peters / CRC Press, 2011.

[28] W. B. Thompson, P. Willemsen, A. A. Gooch, S. H. Creem-Regehr, J. M. Loomis, and A. C. Beall. Does the quality of the computer graphics matter when judging distances in visually immersive environments? Presence: Teleoperators and Virtual Environments, 13(5):560-571, 2004.

[29] M. Usoh, E. Catena, S. Arman, and M. Slater. Using presence questionnaires in reality. Presence: Teleoperators in Virtual Environments, 9(5):497-503, 1999.

[30] P. Willemsen, A. A. Gooch, W. B. Thompson, and S. H. CreemRegehr. Effects of stereo viewing conditions on distance perception in virtual environments. Presence: Teleoperators and Virtual Environments, 17(1):91-101, 2008. 